\title{
Multi-resolution analysis of wavelet transform on pressure fluctuations in an L-valve
}

\author{
T.-Y. Yang, L.-P. Leu* \\ Department of Chemical Engineering, National Taiwan University, 1, Roosevelt Road, Sec. 4, Taipei 10617, Taiwan
}

Received 1 August 2007; received in revised form 19 November 2007

\begin{abstract}
A novel diagnostic method to characterize the flow patterns in an $80 \mathrm{~mm}$-i.d. L-valve had been developed by using multi-resolution analysis (MRA) of wavelet transformation on the pressure fluctuation signals which were acquired from the standpipe and the horizontal part of L-valve. Parameters including the aeration rate, aeration positions, riser gas velocity and composition of binary particle mixture (194- $\mu \mathrm{m}$ and $937-\mu \mathrm{m}$ sand particles) were used to investigate the relationship of performance of L-valve and its pressure fluctuations. By means of MRA, the original pressure fluctuations were divided into multi-scale signals. They were macro-scale, meso-scale and microscale successfully described the structures of gas-solid flow in the L-valve, such as the gas bubbles/slugs, dune-ripple flow, suspension particle flow, etc.
\end{abstract}

(C) 2008 Elsevier Ltd. All rights reserved.

Keywords: L-valve; Pressure fluctuations; Wavelet transform; Multi-resolution analysis

\section{Introduction}

L-valve is one of the most effective and frequently used non-mechanical valve for conveying solid flow in the fluidized bed systems. It mainly consists of a long vertical pipe (standpipe) and a horizontal pipe. The solid flow through the L-valve is controlled by injecting small amount of aeration gas near the bottom of the standpipe. Although its performance, flow dynamic and design procedures had been investigated and reported by some previous studies (Knowlton and Hirsan, 1978; Geldart and Jones, 1991; Ozawa et al., 1991; Loung and Bhattacharya, 1993; Smolders and Baeyens, 1995; Arena et al., 1998), the study of the pressure fluctuations in L-valve for different flow patterns is sparse and the relationship between the standpipe and the L-valve is seldom discussed. Ozawa et al. (1991) measured the fluctuations of static pressure at the lower part

\footnotetext{
${ }^{*}$ Corresponding author. Tel.: +88602 2365 7200; fax: +88602 2362 3040.

E-mail address:1leulii@ntu.edu.tw (L.-P. Leu).
}

of the standpipe. They qualitatively discussed the relationship between the pressure fluctuations and the corresponding flow patterns (packed-bed, coexistence, oscillation and pseudo-bridge patterns) in the standpipe. However, no further discussion of the pressure fluctuations in the horizontal part of L-valve was proposed in their study. To understand the more detail characteristics of different flow patterns in an L-valve, it is necessary to integrate the study of the pressure fluctuations in the standpipe and the horizontal part of L-valve.

In recent years, a powerful signal processing tool, wavelet transform, has been applied to analyze various kinds of signal in scientific and engineering fields. It is especially adequate to deal with a signal which contains multi-scale features and unsteady characteristics, such as the pressure fluctuations in the gas-solid flow systems. So far, it has been shown to provide much significant information from the pressure signals measured in literatures. $\mathrm{Lu}$ and $\mathrm{Li}$ (1999) proposed that wavelet function analyzes the pressure fluctuation signals and indicated the scale 4 detail signal reflects the bubble behaviors in a fluidized bed. Ren and 
Li (1998) used wavelet transform to decompose the original pressure fluctuations into three scales corresponding to macro-, meso- and micro-scales. The first one characterizes the effect of the processing unit on the system behavior, the second one describes the interaction of clusters or bubbles and the third one mainly characterizes particle motion in clusters or dilute phase. They verified that a fluidized bed exhibits multi-scale behaviors causing multi-resolution components in original time series of pressure fluctuations. Park and Kim (2001) used wavelet and Fourier transforms to analyze the pressure fluctuations in a three-phase fluidized bed. They characterized two different flow regimes in the bed by the dominant scale of wavelet coefficients and the highest wavelet energy. Li $(2000,2002)$ employed wavelet multi-resolution analysis and statistic methods (root mean square, skewness factor and probability density function) to the pressure fluctuations of gas-solid flow in a horizontal pipe.

The aim of this paper is to investigate the pressure fluctuations in the standpipe and the horizontal part of the L-valve under different operation conditions by using the multi-resolution analysis (MRA) of wavelet transformation. According to this analytic method, the flow mechanism of the gas-solid flow in the L-valve was characterized by the energy distribution of the multi-scale signals.

\section{Experimental setup and procedure}

Experiments were conducted in a Plexiglas. L-valve which had an 80-mm-i.d. $\times 380$-mm-long horizontal section and its vertical section was connected with an 80mm-i.d. $\times 1760-\mathrm{mm}$ high Plexiglas standpipe. The 52$\mathrm{mm}$-i.d. riser was used for recirculation of solid particles from L-valve to the storage tank equipped at the top of the standpipe. Three aeration taps (labeled as A1, A2 and A3) were located at $0.17 \mathrm{~m}, 0.27 \mathrm{~m}$ and $0.37 \mathrm{~m}$ above the centerline of the horizontal section of the L-valve respectively. Fig. 1 shows the scheme of the experimental setup. Ambient air was supplied by Roots blower to lift the solid particles in the riser. The aeration gas injected into the L-valve was supplied by a compressor and the aeration rate was measured by a rotameter. Two different sizes of sand particles were employed as bed materials and their properties are listed in Table 1. The solid flow rate of the sand particles was obtained by timing the solid particle velocity at the standpipe wall between two marks $(0.1 \mathrm{~m}$ apart) at steady-state operation and calibrated by the solid flow rate determined by collecting the solids in a container over a measured time interval as proposed by Knowlton and Hirsan (1978).

Several pressure taps were installed in $0.1-\mathrm{m}$ interval apart along the horizontal and vertical sections of the Lvalve. To avoid the blockage by fine particles, the tip of each pressure probe was covered with screen of 400 mesh, and was flushed with the inside wall of the L-valve. In each run of experiments, the pressure fluctuation signals were recorded by an $\mathrm{AD} / \mathrm{DA}$ card at a rate of $100 \mathrm{~Hz}$ with

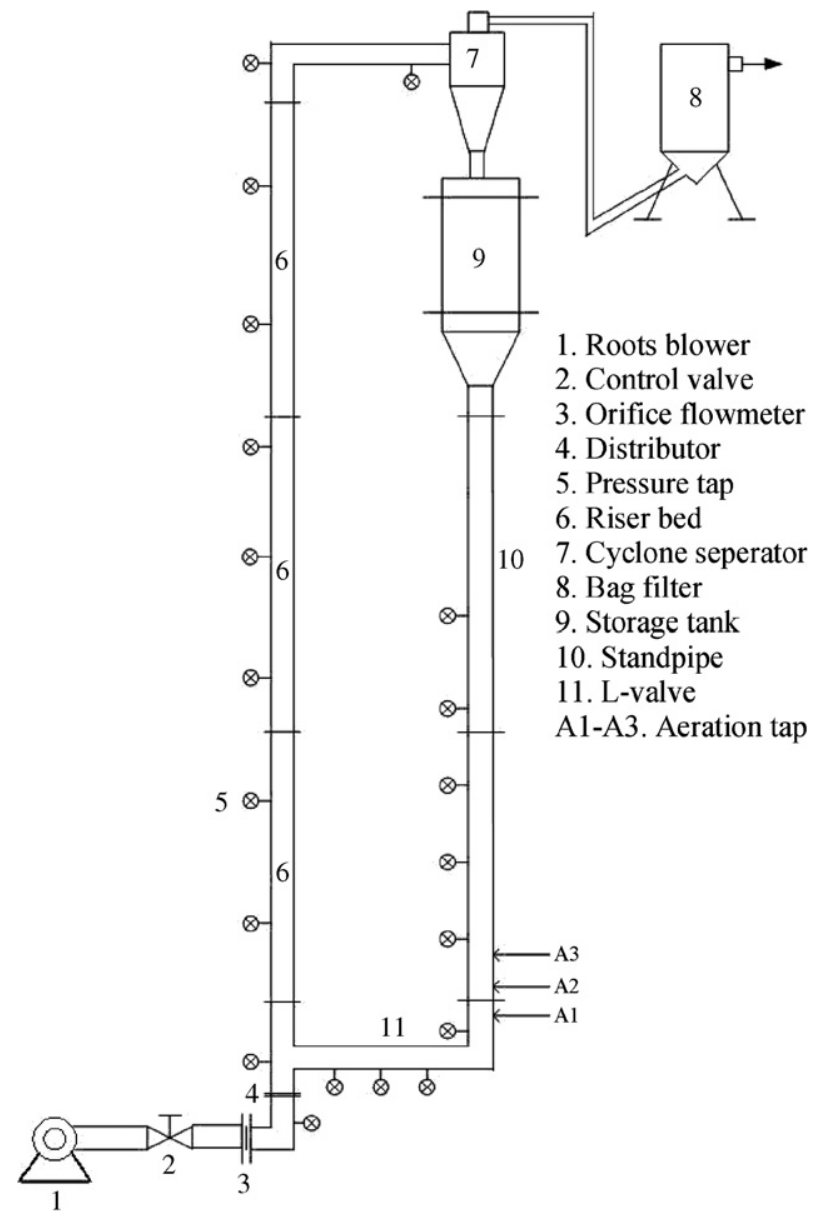

Fig. 1. Experimental setup.

Table 1

Properties of solid particles

\begin{tabular}{lll}
\hline & Geldart's classification & \\
\cline { 2 - 3 } & $B$ & $D$ \\
\hline$d_{\mathrm{p}}(\mu \mathrm{m})$ & 194 & 937 \\
$\rho_{\mathrm{s}}\left(\mathrm{kg} / \mathrm{m}^{3}\right)$ & 2635 & 2635 \\
$U_{\mathrm{mf}}(\mathrm{m} / \mathrm{s})^{\mathrm{a}}$ & 0.0382 & 0.582 \\
$U_{\mathrm{ms}}(\mathrm{m} / \mathrm{s})^{\mathrm{b}}$ & 0.096 & 0.64 \\
\hline
\end{tabular}

${ }^{\mathrm{a}}$ Estimated from Wen and $\mathrm{Yu}(1966)$.

b Estimated from Stewart and Davidson (1967).

$81.92 \mathrm{~s}$ of sampling duration. The multi-resolution analysis of wavelet transformation was carried out off-line by using the wavelet toolkit of S-PLUS software.

\section{Signal processing of experimental data}

\subsection{Wavelet transformation}

Wavelet transform is a relatively novel mathematical tool for signal processing. Similar to a windowed Fourier transform, a wavelet transform can measure the time-frequency variations of spectral components, but it provides 
more flexible time-frequency resolutions. The transform on a discrete signal can be carried out by discrete wavelet transform (DWT). The essence of DWT is to expand a pressure signal, $x(t)(t=1, \ldots, N)$, as a sum of base functions $\phi_{j, k}(t)$ and $\psi_{j, k}(t)$. They are produced by dilations and translations of the orthogonal father wavelet function $\phi$ and the mother wavelet function $\psi$ as follows:

$\phi_{j, k}(t)=2^{-j / 2} \phi\left(\frac{t-2^{j} k}{2^{j}}\right) \quad j, k \in I$

$\psi_{j, k}(t)=2^{-j / 2} \psi\left(\frac{t-2^{j} k}{2^{j}}\right) \quad j, k \in I$

here the time shift $k=1,2, \ldots, N / 2^{j}$ and the level $j=1,2, \ldots, J . J$ is the maximum level of wavelet transform and is dependent on $\phi, \psi$ and $N$. Thus, the wavelet transform of $x(t)$ can be obtained by Eqs. (3) and (4),

$$
\begin{aligned}
& s_{J, k}=\int x(t) \phi_{J, k}(t) \mathrm{d} t \\
& d_{j, k}=\int x(t) \psi_{j, k}(t) \mathrm{d} t
\end{aligned}
$$

where $s_{J, k}$ and $d_{j, k}$ are called the approximation/smooth and detail coefficients respectively. Roughly speaking, $s_{J, k}$ mainly represents the smooth behavior of $x(t)$ at the coarser scale and $d_{j, k}$ represents the detail part at the finer scale. The latter also provides the deviation between two successive scales of smooth coefficients $s_{j, k}$ and $s_{j-1, k}$.

\subsection{Multi-resolution analysis (MRA)}

Multi-resolution analysis, first developed by Mallat (1989), can be applied to decompose the signal $x(t)$ into various scales of orthogonal signal component. They are the approximation subsignal $S_{J}(t)$ and the detail subsignal $D_{j}(t)$, which represent the components of $x(t)$ at different resolutions, calculated as follows:

$$
\begin{aligned}
& S_{J}(t)=\sum_{k} s_{J, k} \phi_{J, k}(t) \quad J, k \in I \\
& D_{j}(t)=\sum_{k} d_{j, k} \psi_{j, k}(t) \quad j, k \in I
\end{aligned}
$$

The $D_{j}(t)$ contents an approximate frequency band of $\left[f_{\mathrm{s}}\right]$ $\left.2^{j+1}-f_{\mathrm{s}} / 2^{j}\right] \mathrm{Hz}$ and the $S_{J}(t)$ contents an approximate frequency band of $\left[0-f_{\mathrm{s}} / 2^{J+1}\right] \mathrm{Hz}$, here $f_{\mathrm{s}}$ is the sampling frequency. Thus, the finer scales of $D_{j}(t)$ mainly capture the detail/high frequency feature of $x(t)$, while the larger scales of $D_{j}(t)$ and $S_{j}(t)$ mainly reveal the whole-view/low-frequency feature of $x(t)$. After that, the original signal $x(t)$ can be recovered in terms of these subsignals with different scales:

$x(t) \approx S_{J}(t)+D_{J}(t)+D_{J-1}(t)+\cdots+D_{1}(t)$
The energy of $S_{J}(t)$ and $D_{j}(t)$ are defined as follows:

$$
\begin{aligned}
& E_{J}^{S}=\sum_{t=1}^{N}\left|S_{J}(t)\right|^{2} \\
& E_{j}^{D}=\sum_{t=1}^{N}\left|D_{j}(t)\right|^{2}
\end{aligned}
$$

So, the energy distribution of $x(t)$ can be calculated by Eqs. (8) and (9) under various levels.

Based on the orthogonality and the energy conservation of wavelet transform, the total energy of $x(t), E$, can be calculated by the sum of $E_{j}^{D}(j=1, \ldots, J)$ and $E_{J}^{S}$ as follows:

$E=\sum_{t}|x(t)|^{2}=E_{J}^{S}+\sum_{j=1}^{J} E_{j}^{D}$

In this study, the third-order Daubechies' wavelet (Daublet3) was used as wavelet function to carryout MRA on the pressure fluctuation signals. The normalized energy of the individual approximation $\bar{e}_{J}^{S}$ and detail subsignals $\bar{e}_{j}^{D}$ can be calculated as follows:

$\bar{e}_{J}^{S}=\frac{E_{J}^{S}}{E}$

$\bar{e}_{j}^{D}=\frac{E_{j}^{D}}{E}$

\section{Results and discussion}

\subsection{Observation of the flow patterns and pressure fluctuations}

In this study, the pressure fluctuation signals were measured simultaneously at the center of standpipe $(z=0.67 \mathrm{~m})$ and the horizontal portion of L-valve. As shown in Table 2, the pressure fluctuations appeared to change obviously as increasing the aeration rate $Q_{\mathrm{a}}$ in the L-valve. Meanwhile, under different $Q_{\mathrm{a}}$ s, three kinds of flow patterns were observed respectively in the standpipe and the horizontal part of L-valve. They were the packed-bed flow (PBF), fluidized-bed flow (FBF) and slugging-bed flow (SBF) patterns for the standpipe; and the fast-moving stream flow (FMSF), dune-ripple flow (DRF), and duneslug flow (DSF) patterns for the horizontal portion of the L-valve. Some similar phenomena had also been observed by the others (Leung and Jones, 1978; Geldart and Jones, 1991; Ozawa et al., 1991; Knowlton, 1997).

\subsection{1. $P B F$ and FMSF patterns}

At the low aeration rate $Q_{\mathrm{a}}^{*}<Q_{\mathrm{a}}<Q_{\mathrm{mf}}$, where $Q_{\mathrm{a}}^{*}$ was the threshold aeration and $Q_{\mathrm{mf}}$ was the aeration rate based on minimum fluidization velocity $U_{\mathrm{mf}}$, the solid particles flowed smoothly downward with no bubbles in the standpipe. This kind of flow was commonly referred to as the packed-bed/moving-bed flow (PBF) (Knowlton and Hirsan, 1978). The corresponding pressure fluctuations had a small amplitude and high frequency component. It repre- 
Table 2

Flow patterns and the pressure fluctuations in the standpipe and the L-valve

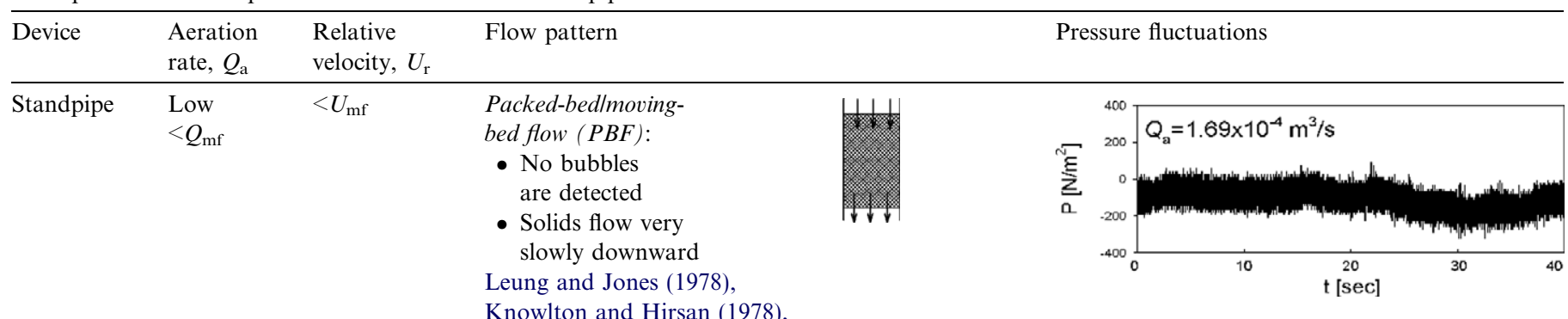

Knowlton and Hirsan (1978),

Jones and Leung (1985),

and Ozawa et al. (1991)

$\underset{>}{\text { Medium }}>U_{\mathrm{mf}}$

$>Q_{\mathrm{mf}}$

High $>U_{\mathrm{ms}}$

$>Q_{\mathrm{ms}}$

Slugging-bed flow (SBF):

- Large slug/cavity are formed near the aeration tap

- Solid flow is hindered and relatively instable

- Pseudo-bridge pattern is observed

Jones and Leung (1985)

Fast-moving stream flow (FMSF):

- Most of solid particles are stagnant, only a suspension particle stream moves fast at the top of horizontal part

Geldart and Jones (1991)

Dune-ripple flow (DRF):

Medium $>U_{\mathrm{mf}}$ $>Q_{\mathrm{mf}}$

- Dunes and ripples move continuously and periodically along the top of horizontal part

- Fluctuations of solid discharge rate and pressure measured just above the aeration position is observed

Geldart and Jones (1991)

High $\quad>U_{\mathrm{ms}}$ $>Q_{\mathrm{ms}}$
Dune-slug flow (DSF):

- Highly instable, solid flow is hindered seriously

- Large dunes and cavities coexist and flow out irregularly
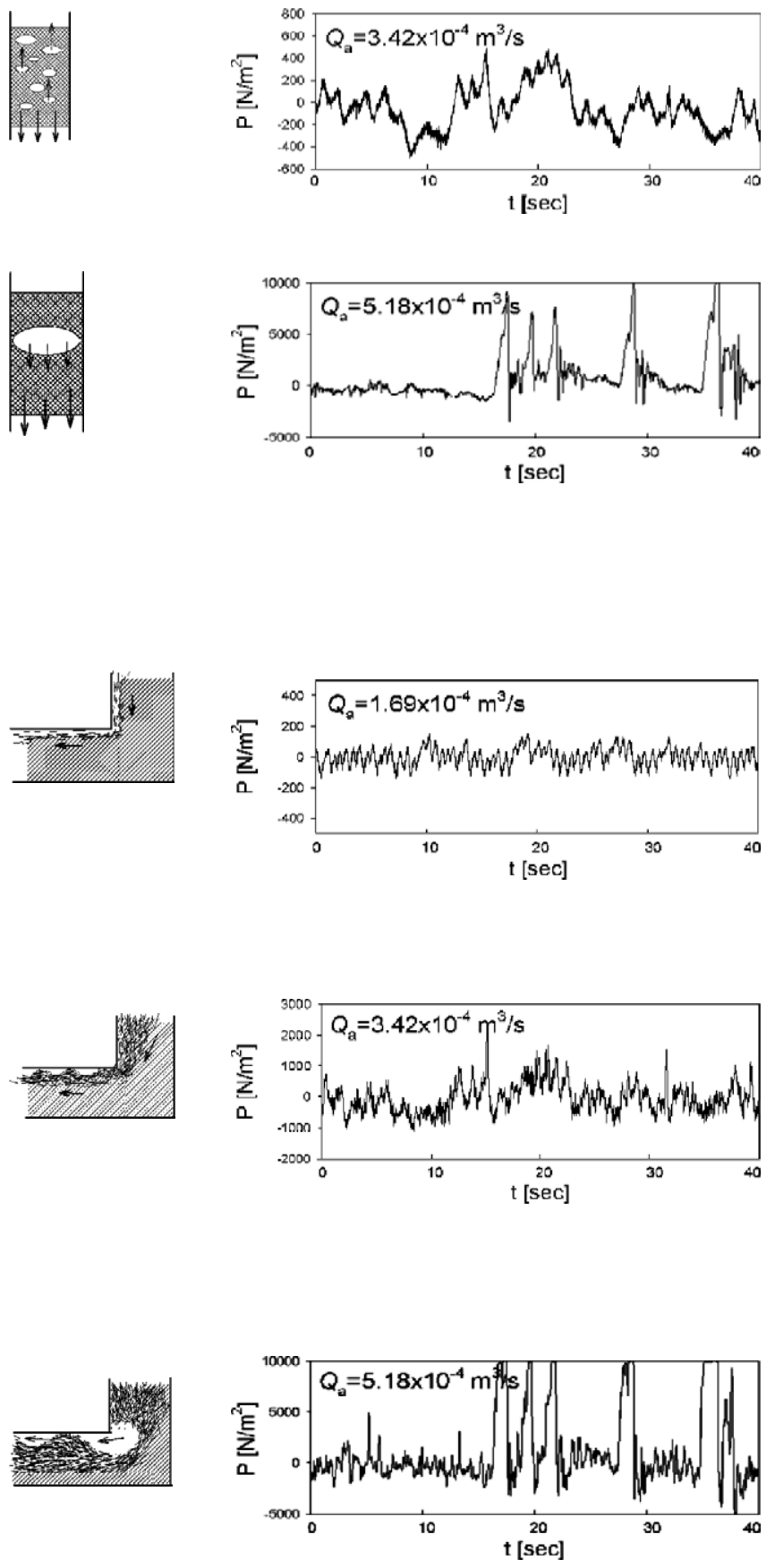
sented that the flow pattern in the standpipe was mainly predominated by stable solid particles flow. Simultaneously, a fast-moving stream flow (FMSF) is observed at the narrow top of horizontal portion of L-valve (Geldart and Jones, 1991). It produces small and regular pressure fluctuations caused by the small ripples periodically entrained with the solid particles stream.

\subsubsection{FBF and DRF patterns}

At the medium aeration rate $Q_{\mathrm{mf}}<Q_{\mathrm{a}}<Q_{\mathrm{ms}}$, where $Q_{\mathrm{ms}}$ was the aeration rate based on incipient slugging velocity $U_{\mathrm{ms}}$, a part of the aeration gas formed small bubbles with frequency about $0.5-1.5 \mathrm{~Hz}$ and flowed upward in the standpipe that somewhat hindered the solid flow and produced larger fluctuations. Because the solid particles were fluidized in the standpipe, so the flow pattern was regarded as the fluidized-bed flow (FBF). On the other hand, the else part of aeration gas was entrained downward by solid flow into the horizontal section of the L-valve. It formed lager ripples and dunes at the inner corner of the bend that produced the pressure fluctuations of higher amplitude. This kind of flow pattern consequently was regarded as the dune-ripple flow (DRF).

\subsubsection{SBF and DSF patterns}

At the high aeration rate $Q_{\mathrm{a}}>Q_{\mathrm{ms}}$, the large slugs/cavities began to develop at the upper section of the standpipe and its size enlarged gradually as moving downward with the solid flow. Once it reached the inner corner of the bend, it was pushed by the large dunes rapidly through the Lvalve. Because the clear slugs/cavities were observed in the standpipe, thus the flow pattern was regarded as the slugging-bed flow (SBF). It was very similar with the "pseudo-bridge" pattern observed by Jones and Leung (1985) who demonstrated that this flow pattern may form as the position of aeration at the standpipe is too high. In the horizontal portion of the L-valve, the corresponding flow pattern was regarded as the dune-slug flow (DSF). The solid flow was highly instable due to the hindrance of the large slugs/cavities. That occasionally caused huge fluctuations of pressure signals both measured at the standpipe and L-valve. The rest part of pressure fluctuations was analogous to that of the DRF pattern with higher amplitude.

\subsection{Multi-resolution analysis (MRA) of pressure fluctuations}

To further understand the characteristics of pressure fluctuations in the L-valve, the MRA of wavelet transform was applied under various flow patterns introduced in the last section. The decomposition level of MRA was set as eight, thus each pressure fluctuation signal was decomposed into nine subsignals. In this case, eight detail subsignals $\left(D_{1}-D_{8}\right)$ and one approximation subsignal $\left(S_{8}\right)$ were obtained from each pressure fluctuation signal under the six flow patterns in the standpipe and the horizontal part
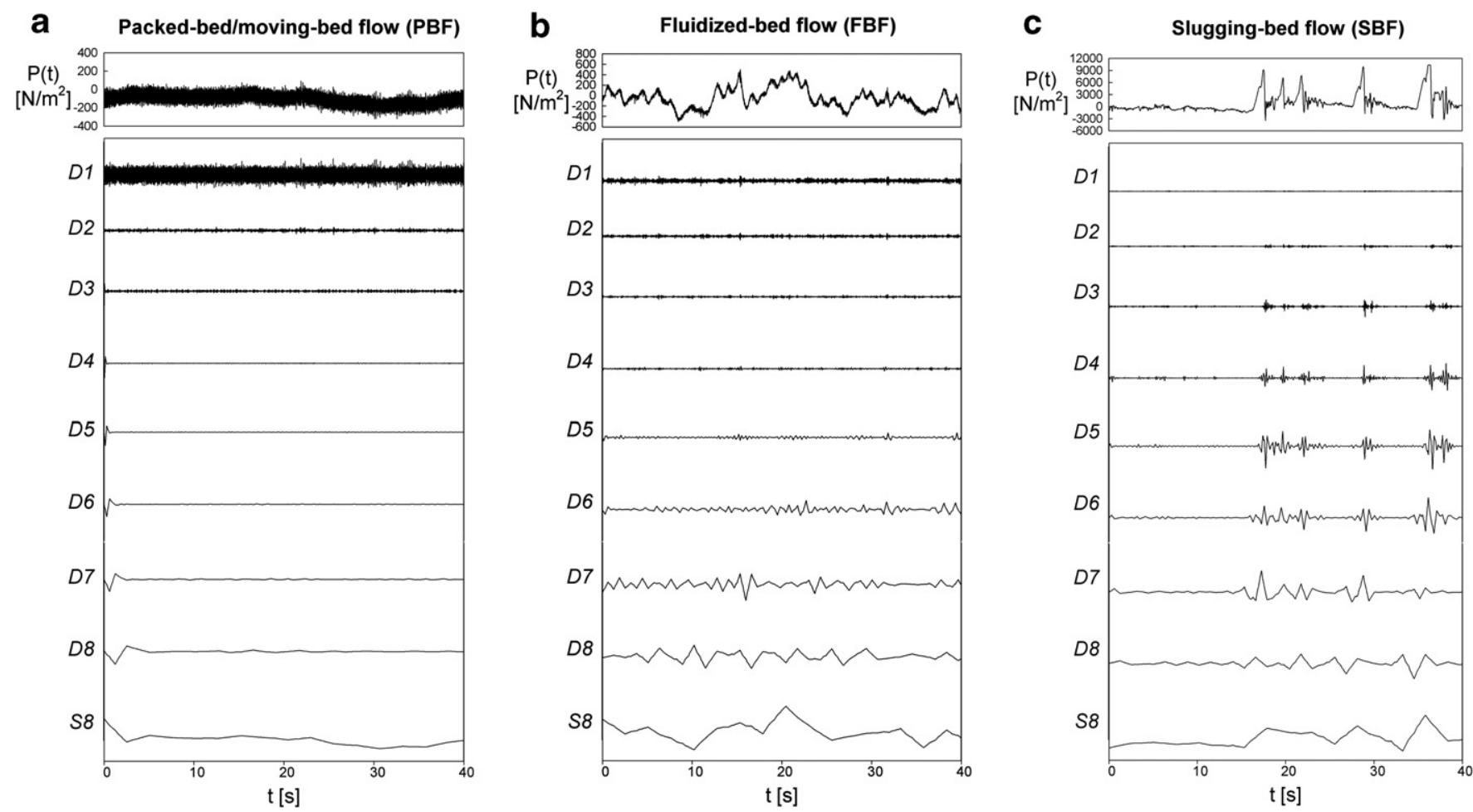

Fig. 2. Multi-resolution analysis of pressure fluctuations in the standpipe at (a) $Q_{\mathrm{a}}=1.69 \times 10^{-4} \mathrm{~m}^{3} / \mathrm{s}, \quad$ (b) $Q_{\mathrm{a}}=3.42 \times 10^{-4} \mathrm{~m}^{3} / \mathrm{s}$, and $(\mathrm{c})$ $Q_{\mathrm{a}}=5.18 \times 10^{-4} \mathrm{~m}^{3} / \mathrm{s}$. 

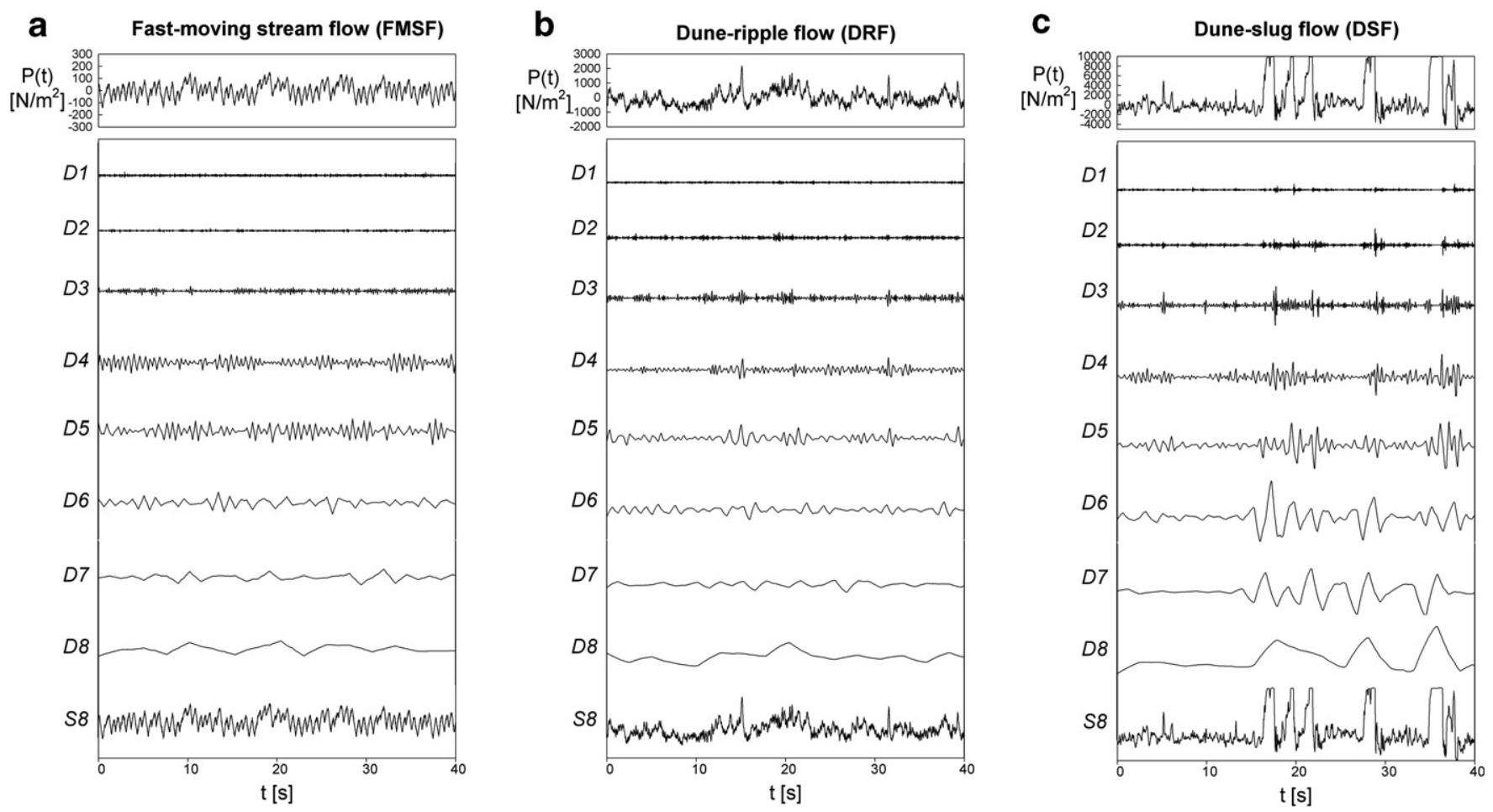

Fig. 3. Multi-resolution analysis of pressure fluctuations in the horizontal part of L-valve (a) $Q_{\mathrm{a}}=1.69 \times 10^{-4} \mathrm{~m}^{3} / \mathrm{s}$, (b) $Q_{\mathrm{a}}=3.42 \times 10^{-4} \mathrm{~m}^{3} / \mathrm{s}$, and (c) $Q_{\mathrm{a}}=5.18 \times 10^{-4} \mathrm{~m}^{3} / \mathrm{s}$.

of L-valve, as shown in Figs. 2a-c and 3a-c, respectively. For the purpose of comparison, each subsignal was plotted as the same scale of coordinates as the original pressure fluctuations. These figures simultaneously provided the information of the fluctuating data in frequency and time domains. Furthermore, the multi-scale behavior of original pressure fluctuations was characterized by recombination of these subsignals. The micro-scale behavior of pressure fluctuations was mainly presented by the finer scales of MRA, e.g. $D_{1}$ and $D_{2}$, which had the frequency bandwidth of $\left[f_{\mathrm{s}} / 8-f_{\mathrm{s}} / 2\right]=[12.5-50] \mathrm{Hz}$ as discussed before. These scales were relatively significant in the PBF pattern. The meso-scale behavior corresponding to the small bubbles and dunes/ripples in the standpipe and L-valve respectively was mainly captured by the detail subsignals $D_{3}, D_{4}, D_{5}$ and $D_{6}$ with the frequency bandwidth of $[0.78-12.5] \mathrm{Hz}$. The macro-scale behavior corresponding to the large slugs/cavities and instable pressure oscillation was described by $D_{7}, D_{8}$ and $S_{8}$ with extremely low-frequency range of $[0-0.78] \mathrm{Hz}$. The amplitude of these subsignals would be expected to increase remarkably in SBF and DSF patterns. Fig. 4 shows the result of multi-scale analysis on the pressure fluctuations in the standpipe and the horizontal part of L-valve.

\subsection{Energy distribution of multi-scale signals}

The energy distribution helped us to realize which subsignal was dominant in the corresponding flow pattern.
Fig. 5 shows the energy distribution of MRA calculated from Eqs. (8) and (9) in various flow patterns. It was evident that whether in the standpipe or the horizontal part of L-valve, $S_{8}$ often had a largest energy. That was contributed to the global pressure drop oscillations or the instable solid mass flow in the entire system. Jones and Leung (1985) also introduced that a jerky flow mode, so-called the slip-stick flow, is observed in the standpipe. It oscillates between flow and no-flow at a frequency in the range of approximately $0.1-1 \mathrm{~Hz}$ which is well consistent with the frequency bandwidth of $S_{8}$ subsignal in this study. In PBF pattern, $D_{1}$ usually had higher energy than any of other detail subsignals, however, it was relative small in the other flow patterns. It was because that $D_{1}$ subsignal with highest frequency content was mainly contributed from the background noise and the fluctuations of interstitial gas flow in the packed-bed. When $Q_{\mathrm{a}}$ was increased, $D_{1}$ would be covered by the other detail subsignals of larger scale which was produced by bubbles or big voids. Furthermore, in the horizontal part of L-valve, the energy of meso-scale subsignals $\left(D_{3}-D_{6}\right)$ played an important role due to the dunes and ripples flow patterns predominated here.

To quantitatively investigate the effect of operation parameters on the pressure fluctuations, the energy of micro-, meso- and macro-scale signals were calculated directly by the sum of corresponding energy of the subsignals $\left(D_{1}-D_{8}\right.$ and $\left.S_{8}\right)$ based on energy conservation. Therefore, from Eq. (10) we had, 

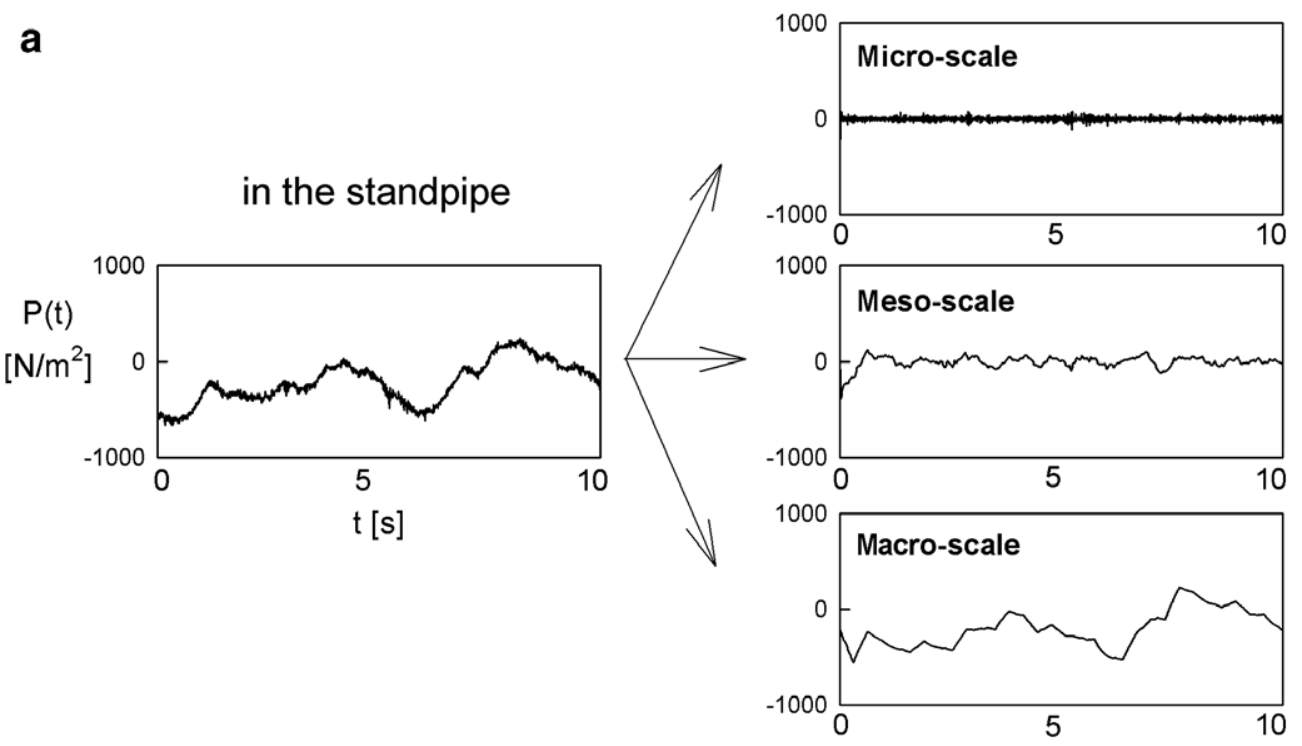

b
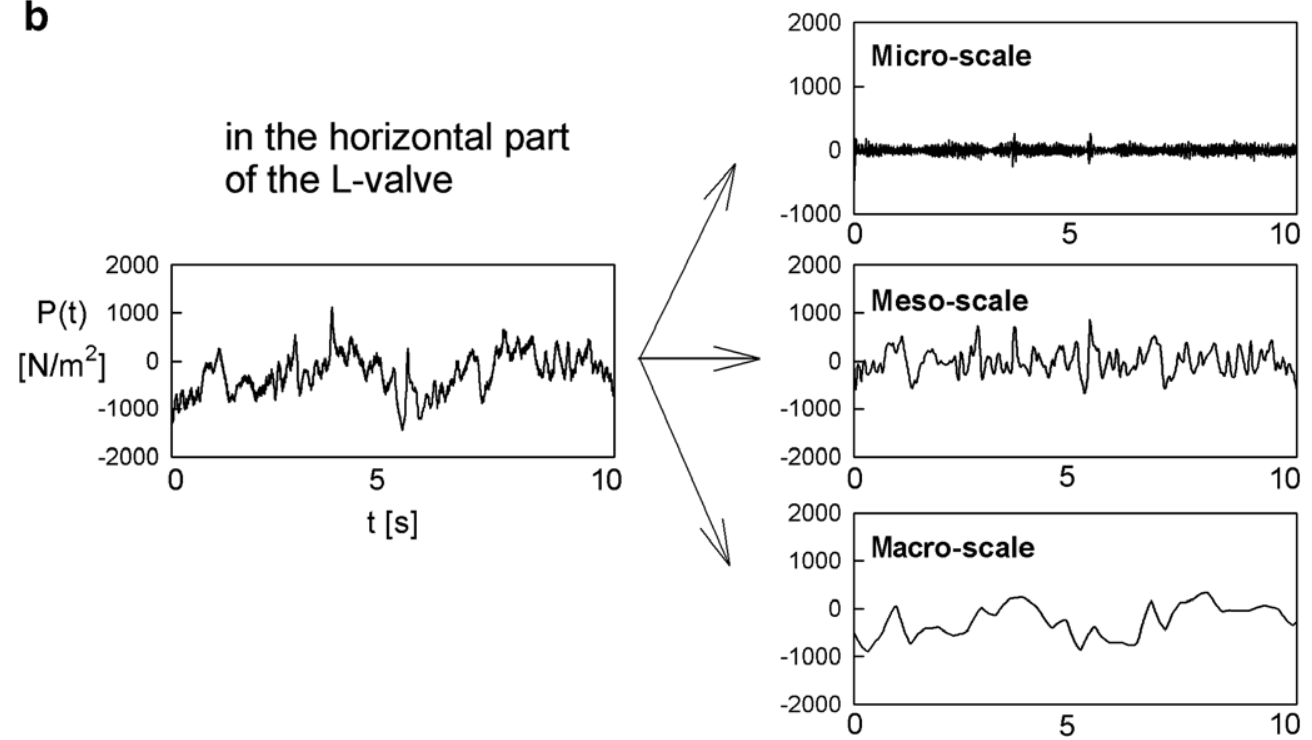

Fig. 4. Multi-scale of pressure fluctuations in the (a) standpipe and (b) horizontal part of L-valve at $U_{\mathrm{g}}=7.22 \mathrm{~m} / \mathrm{s}, Q_{\mathrm{a}}=4.00 \times 10^{-4} \mathrm{~m}^{3} / \mathrm{s}$.

$E=\sum_{t}|x(t)|^{2}=\sum_{j=1}^{8} E_{j}^{D}+E_{8}^{S}=E_{\text {micro }}+E_{\text {meso }}+E_{\text {macro }}$

$E_{\text {micro }}=E_{1}^{D}+E_{2}^{D}$

$E_{\text {meso }}=E_{3}^{D}+E_{4}^{D}+E_{5}^{D}+E_{6}^{D}$

$E_{\text {macro }}=E_{7}^{D}+E_{8}^{D}+E_{8}^{S}$

In addition, the normalized energy $\overline{E_{i}}$ of each scale of signal was obtained as follows:

$\overline{E_{i}}=E_{i} / E, \quad i=$ micro, meso, macro

\subsection{Effect of the aeration rates $Q_{a}$}

Fig. 6 shows the normalized energy of micro-, meso- and macro-scale signals ( $\bar{E}_{\text {micro }}, \bar{E}_{\text {meso }}$ and $\left.\bar{E}_{\text {macro }}\right)$, deduced from
MRA, as a function of $Q_{\mathrm{a}}$ in different flow patterns. It was evident that the multi-scale signals strongly depended on $Q_{\mathrm{a}}$. In the standpipe (Fig. 6a), the most of energy was concentrated in the macro-scale signal with relatively larger amplitude. The micro-scale signal had higher energy content than the meso-scale signal in the PBF pattern where almost no bubbles were observed in the standpipe. In the FBF pattern $\bar{E}_{\text {micro }}$ decreased apparently with the increase of $Q_{\mathrm{a}}$, on the contrary, $\bar{E}_{\text {meso }}$ first increased slightly with the increase of $Q_{\mathrm{a}}$, and then dramatically increased as $Q_{\mathrm{a}}$ approached $Q_{\mathrm{ms}}$. It provided that the bubbles flowed upwards and grew in the standpipe at the relatively higher $Q_{\mathrm{a}}$. In the horizontal part of L-valve (Fig. 6b), $\bar{E}_{\text {meso }}$ appeared to be higher than that in the standpipe. It represented that the small dunes and ripples dominated at low aeration (FMSF pattern), however it was rapidly surpassed by $\bar{E}_{\text {macro }}$ due to the increase of solid flow oscillations 

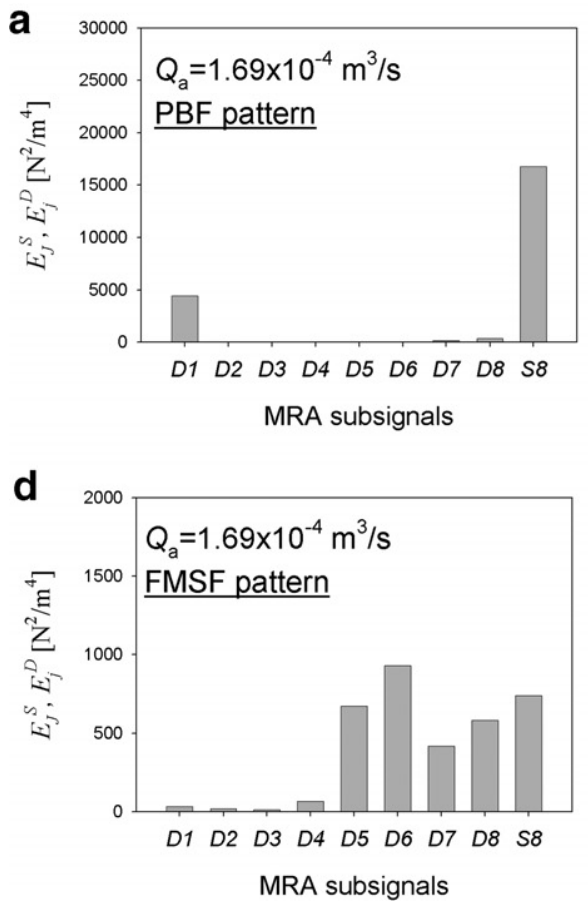
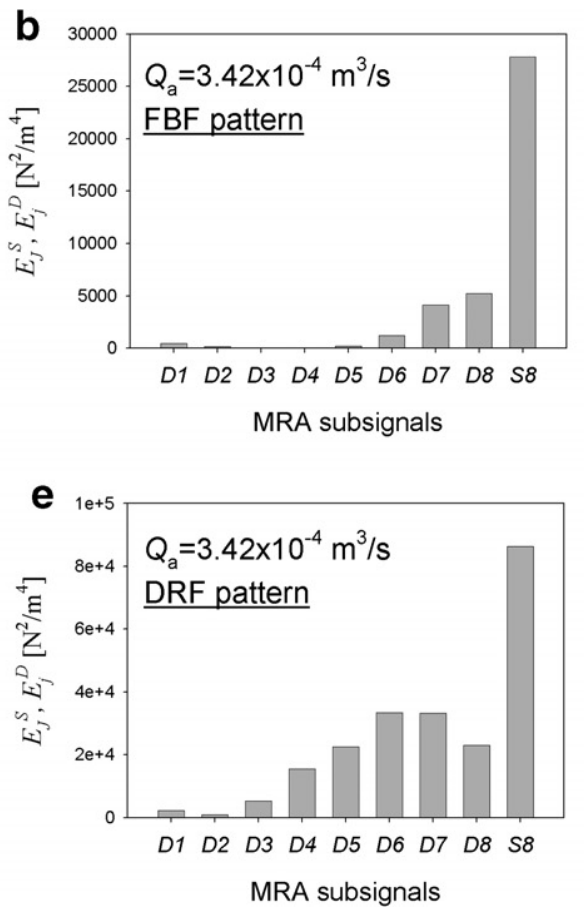
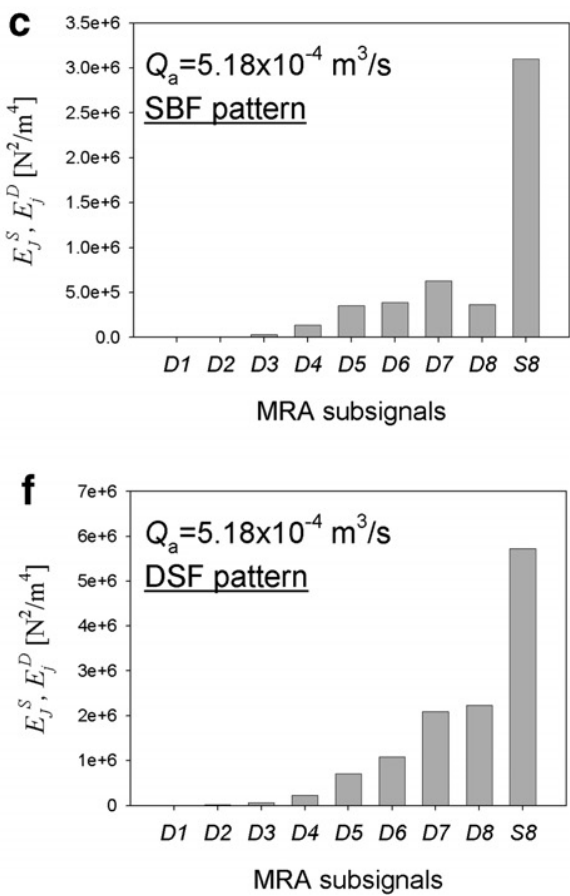

Fig. 5. Energy of MRA subsignals in various flow patterns.

caused by the formation of large bubbles/cavities. At the medium $Q_{\mathrm{a}}$ (DRF pattern), $\bar{E}_{\text {meso }}$ increased smoothly with increasing $Q_{\mathrm{a}}$ then decreased as the large and instable slugs/cavities formed at the higher $Q_{\mathrm{a}}$ (DSF pattern). $\bar{E}_{\text {micro }}$ was almost independent of $Q_{\mathrm{a}}$ and was of little significance in the horizontal part of L-valve where the dunes and ripples of larger scale predominated.

\subsection{Effect of the aeration positions (A1, A2, and A3)}

Fig. 7 shows the dependency of $G_{\mathrm{s}}$ on $Q_{\mathrm{a}}$ with the aeration positions (A1, A2, and $\mathrm{A} 3$ ) as a parameter. It was found that with increasing the aeration gas the difference of $G_{\mathrm{s}}$ among $\mathrm{A} 1, \mathrm{~A} 2$, and $\mathrm{A} 3$ was not obvious for the low and medium $Q_{\mathrm{a}}$. However, as $Q_{\mathrm{a}}$ was increased near $Q_{\mathrm{ms}}\left(4.83 \times 10^{-4} \mathrm{~m}^{3} / \mathrm{s}\right.$ for $194-\mu \mathrm{m}$ sand particles in this study), the difference appeared to be noticeable. Beyond $Q_{\mathrm{ms}}$, the deviations between $\mathrm{A} 1, \mathrm{~A} 2$ and A3 gradually increased with increasing $Q_{\mathrm{a}}$. In addition $G_{\mathrm{s}}$ for A3 did not increase with increasing $Q_{\mathrm{a}}$, but decreased instead. The similar results were also found by Knowlton and Hirsan (1978), who attributed the decreasing of $G_{\mathrm{s}}$ at higher aeration position to the decreasing of effective downcomer/standpipe length. Ozawa et al. (1991) observed the formation of bubbles and cavity in the standpipe when the aeration tap was relative high. Their static pressure data in the standpipe exhibit high amplitude with low-frequency fluctuations and $G_{\mathrm{s}}$ is much smaller than that of the lower aeration tap.

Fig. 8 shows the normalized energy of subsignals $D_{1}-D_{8}$ and $S_{8}$, obtained from Eqs. (11) and (12), for different aeration positions. In PBF and FBF pattern (Figs. 8a and b), the energy distributions of $\mathrm{A} 1, \mathrm{~A} 2$, and $\mathrm{A} 3$ were quite similar. While in the SBF pattern (Fig. 8c), the energy of $D_{4}$, $D_{5}$, and $D_{6}$ at higher aeration positions (A2 and A3) was much larger than that for A1. It revealed that considerable amount of bubbles appeared in the standpipe at the higher aeration positions and hindered the solid flow. In the horizontal part of L-valve, there was no significant effect of the aeration positions on the energy distribution of MRA signals (Figs. 8d-f).

Fig. 9 shows $\bar{E}_{\text {micro }}, \bar{E}_{\text {meso }}$ and $\bar{E}_{\text {macro }}$ as the functions of the aeration positions in the same operational conditions in Fig. 8. In each of flow pattern, $\bar{E}_{\text {micro }}$ was almost independent of the aeration positions. $\bar{E}_{\text {meso }}$ at the PBF and FBF patterns in the standpipe had relative small values and showed almost no difference among the three aeration positions (Figs. 9a and b). The effect of aeration positions on these flow patterns was not significant in the standpipe. However, when $Q_{\mathrm{a}}$ was increased to $5.18 \times 10^{-4} \mathrm{~m}^{3} / \mathrm{s}$, at which the SBF pattern was presented, $\bar{E}_{\text {meso }}$ appeared to increase with heightening the aeration position (Fig. 9c). It indicated that the interference from bubbles flowing upwards in the standpipe became more significant at the higher aeration position. In the horizontal part of the L-valve, the effect on $\bar{E}_{\text {micro }}$ also was insignificant. $\bar{E}_{\text {meso }}$, at the FMSF and DRF patterns, increased slightly with heightening the aeration position (Figs. 9d and e). As $Q_{\mathrm{a}}$ was increased beyond $Q_{\mathrm{ms}}, \bar{E}_{\text {meso }}$ attenuated to a lower value $(<0.2)$ and also slightly decreased with heightening the aeration position (Fig. 9f). In summary, the aeration positions showed significant effect on $\bar{E}_{\text {meso }}, \bar{E}_{\text {macro }}$ and $G_{\mathrm{s}}$ only at relatively higher $Q_{\mathrm{a}}$ in the standpipe. 

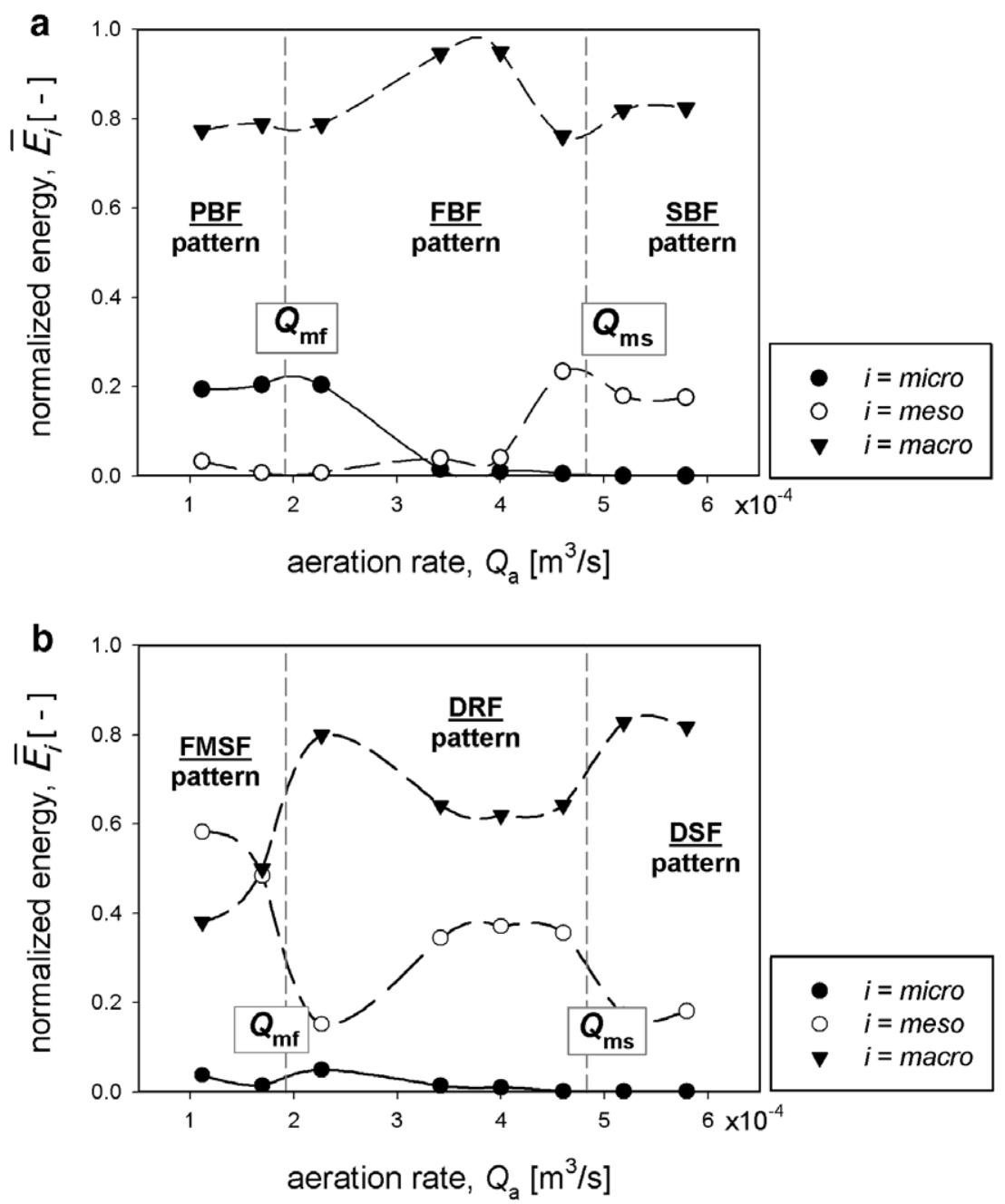

Fig. 6. Normalized energy of micro-, meso- and macro-scale signals in the (a) standpipe and (b) horizontal part of L-valve at $U_{\mathrm{g}}=7.22 \mathrm{~m} / \mathrm{s}$.

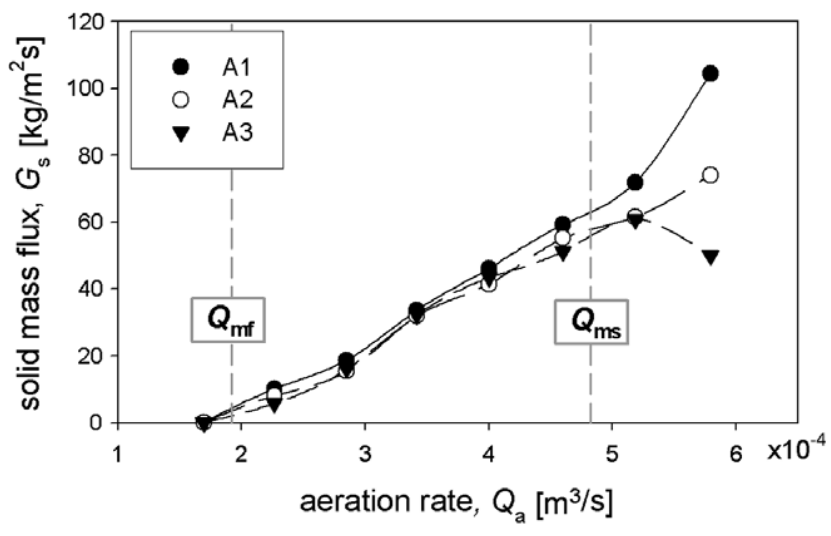

Fig. 7. Effect of aeration positions on solid mass flux at $U_{\mathrm{g}}=7.22 \mathrm{~m} / \mathrm{s}$.

\subsection{Effect of the riser gas velocities $U_{g}$}

Fig. 10 shows the solid mass flux $G_{\mathrm{s}}$ versus the riser gas velocities $U_{\mathrm{g}}$ with the aeration rates as the parameter. For lower aeration rate $\left(Q_{\mathrm{a} 1}\right.$ and $\left.Q_{\mathrm{a} 2}\right), G_{\mathrm{s}}$ did not change obviously with increasing $U_{\mathrm{g}}$. However, for higher aeration rates $\left(Q_{\mathrm{a} 3}-Q_{\mathrm{a} 5}\right) G_{\mathrm{s}}$ increased remarkably with increasing $U_{\mathrm{g}}$. This phenomenon was in agreement with a previous study by Loung and Bhattacharya (1993). Besides, if $Q_{\mathrm{a}}$ was too high $\left(5.79 \times 10^{-4} \mathrm{~m}^{3} / \mathrm{s}\right.$ in this study), the solid flow in the L-valve was obviously hindered for the lower $U_{\mathrm{g}}$. As at $U_{\mathrm{g}}=6.23 \mathrm{~m} / \mathrm{s}$ in Fig. $10, G_{\mathrm{s}}$ of $Q_{\mathrm{a} 5}$ curve was even lower than that of $Q_{\mathrm{a} 3}$ and $Q_{\mathrm{a} 4}$. It was found that increasing $U_{\mathrm{g}}$ would be contributive to eliminate the hindrance to the solid flow.

The comparison of energy distribution at the different $U_{\mathrm{g}}$ is shown in Fig. 11. For relatively higher aeration rate, as decreasing the riser velocity $U_{\mathrm{g}}$, the energy distribution both in the standpipe and the horizontal part of L-valve gradually shifted from the medium level $\left(D_{4}, D_{5}, D_{6}\right)$ to the higher level $\left(D_{8}\right.$ and $\left.S_{8}\right)$ of subsignals. It indicated the L-valve was primarily occupied by low-frequency slugs and cavities with large amplitude at the lower $U_{\mathrm{g}}$. On the other hand, increasing the $U_{\mathrm{g}}$ was helpful to eliminate large slugs/cavities and enlarged the operation range of the L-valve. Fig. 12 shows that $\bar{E}_{\text {macro }}$ decreased apparently as expected with increasing $U_{\mathrm{g}}$ in the riser bed. It 

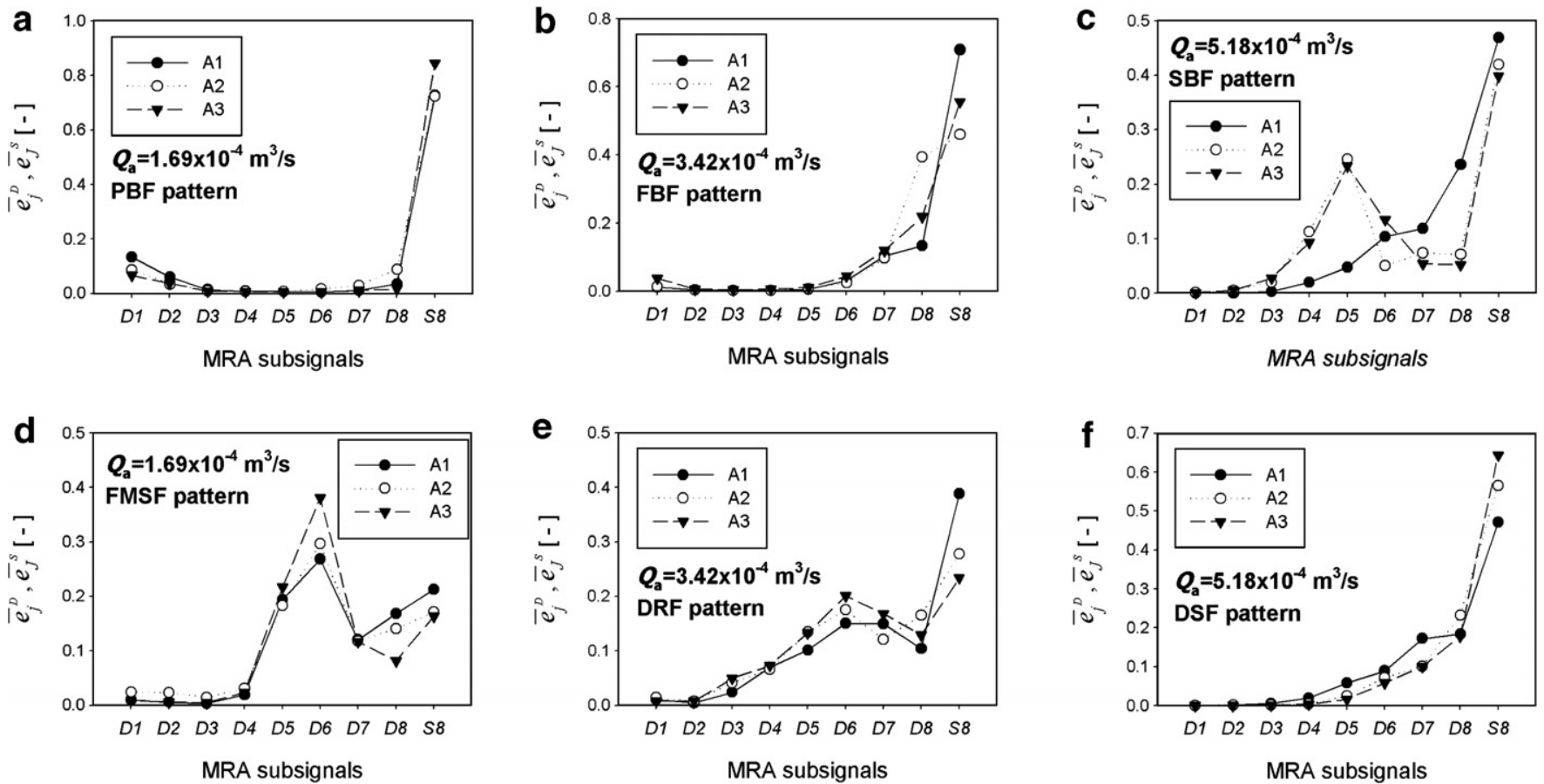

Fig. 8. Normalized energy distribution of MRA subsignals for different aeration positions.
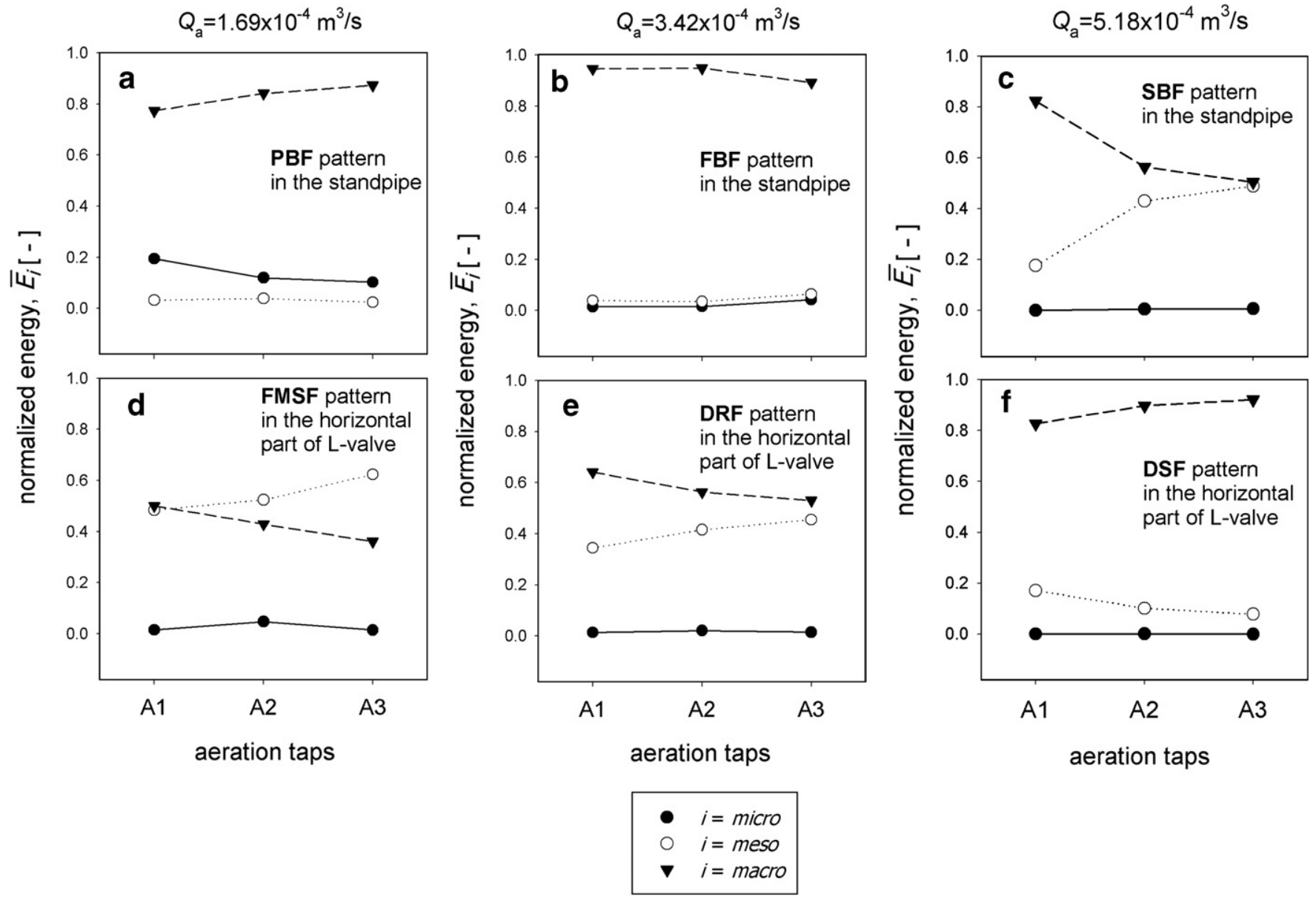

Fig. 9. Normalized energy of micro-, meso- and macro-scale signals in the standpipe and the horizontal part of L-valve for different aeration positions 


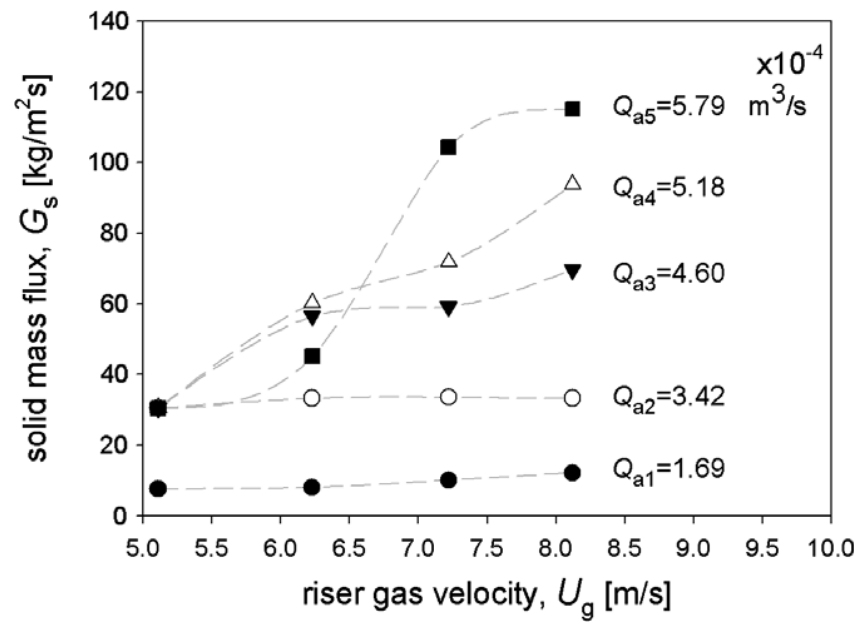

Fig. 10. Solid mass flux versus riser velocities with different aeration rates.
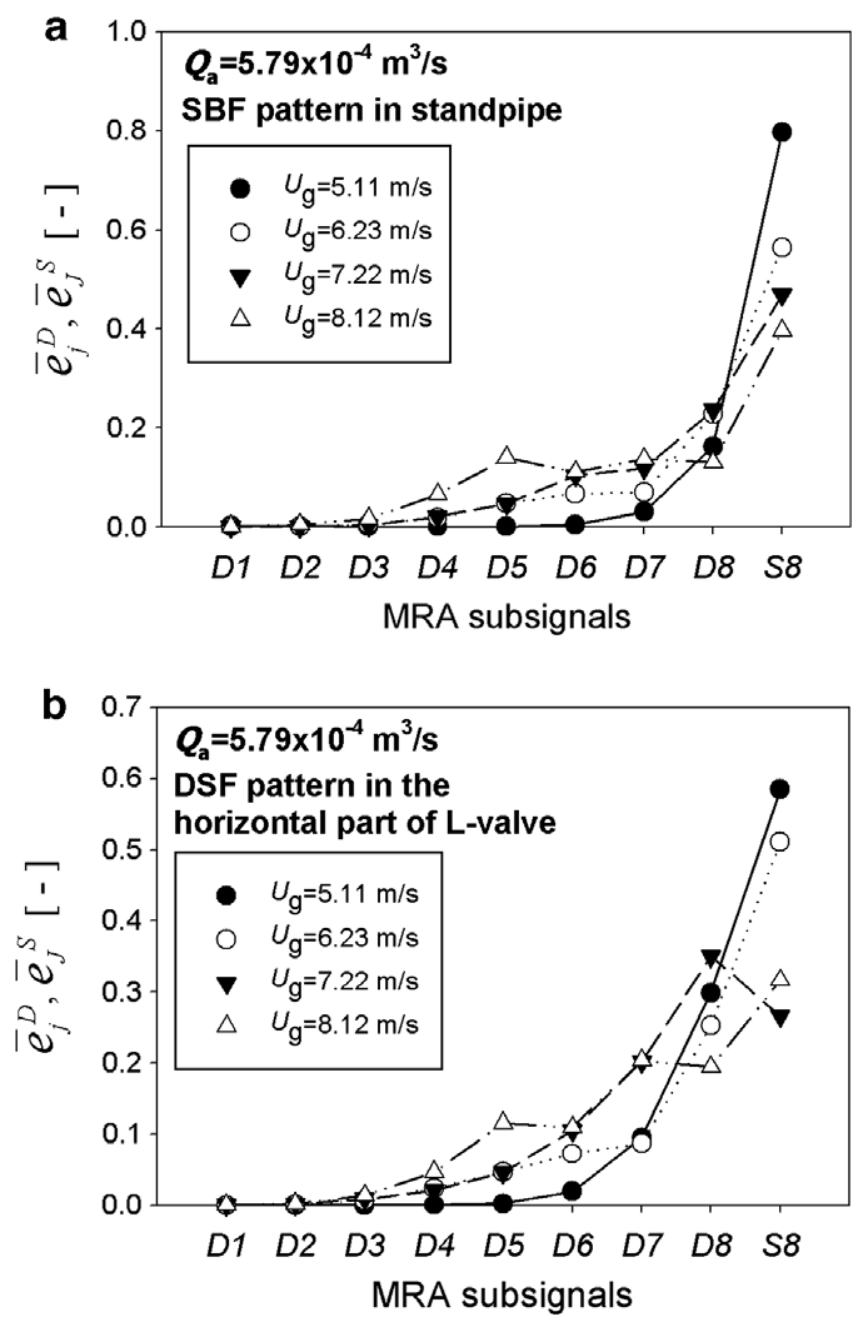

Fig. 11. Normalized energy distribution of MRA subsignals at different riser velocities.

meant that the large slugs/cavities split into smaller bubbles/gas void and $\bar{E}_{\text {meso }}$ consequently increased with increasing $U_{\mathrm{g}}$.
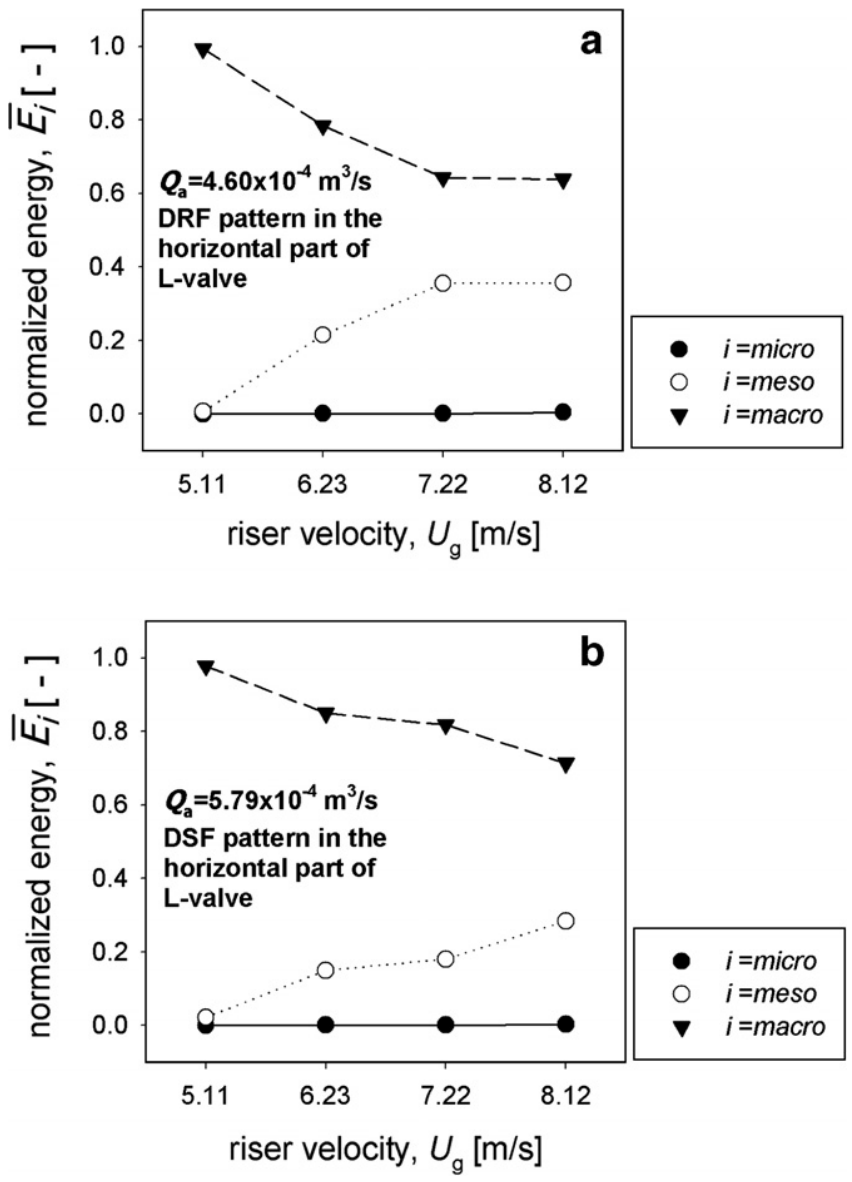

Fig. 12. Dependency of normalized energy of micro-, meso- and macroscale signals on the $U_{\mathrm{g}}$ for (a) $Q_{\mathrm{a}}=4.60 \times 10^{-4} \mathrm{~m}^{3} / \mathrm{s}$ and (b) $Q_{\mathrm{a}}=5.79 \times 10^{-4} \mathrm{~m}^{3} / \mathrm{s}$.

Table 3

Properties of binary particle mixture

\begin{tabular}{lllll}
\hline & \multicolumn{4}{l}{ Composition of mixtures $\left(x_{\mathrm{B}}: x_{\mathrm{D}}\right)$} \\
\cline { 2 - 5 } & $8: 2$ & $7: 3$ & $6: 4$ & $5: 5$ \\
\hline$d_{\mathrm{p}}(\mu \mathrm{m})$ & 231 & 254 & 284 & 321 \\
$\rho_{\mathrm{s}}\left(\mathrm{kg} / \mathrm{m}^{3}\right)$ & 2635 & 2635 & 2635 & 2635 \\
$U_{\mathrm{mf}}(\mathrm{m} / \mathrm{s})^{\mathrm{a}}$ & 0.0426 & 0.0488 & 0.0591 & 0.0755 \\
$U_{\mathrm{ms}}(\mathrm{m} / \mathrm{s})^{\mathrm{b}}$ & 0.105 & 0.111 & 0.121 & 0.138 \\
\hline \multicolumn{5}{c}{ astimated from Cheung et al. (1974). } \\
\multicolumn{2}{c}{ b Estimated from Stewart and Davidson (1967). }
\end{tabular}

\subsection{Effect of the compositions of binary particle mixture}

In order to understand the effect of the compositions of binary particle mixture on the performance of L-valve, 194$\mu \mathrm{m}$ and $937-\mu \mathrm{m}$ sand particles were mixed up in four specific compositions. Some of their properties are listed in Table 3.

In Fig. 13, $G_{\mathrm{s}}$ for each binary particle mixture pair increased linearly with increasing the aeration number $U /$ $U_{\mathrm{mf}}$, here $U$ was the superficial gas velocity based on the cross-sectional area of the standpipe/L-valve. In addition, the workability range (i.e. the difference between the 


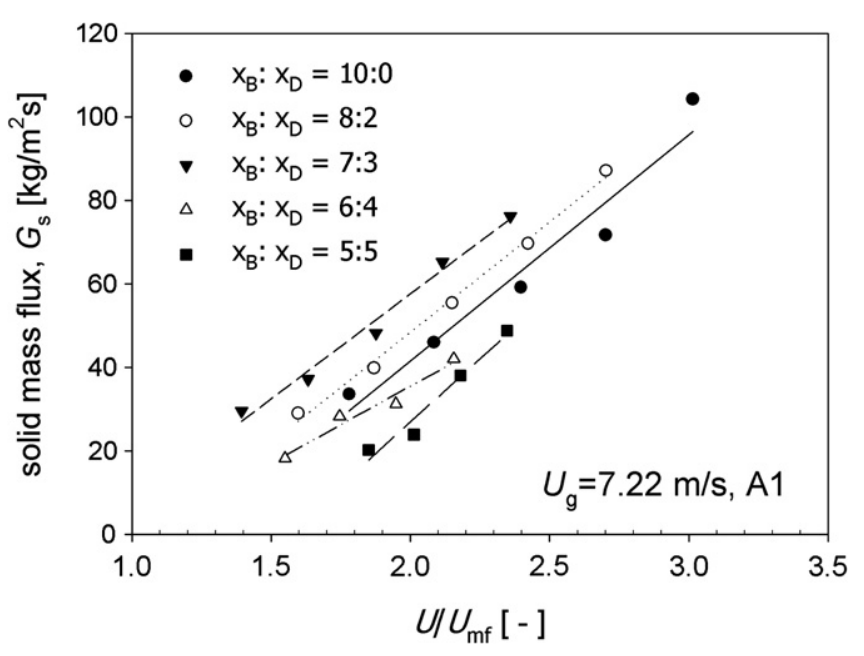

Fig. 13. Solid mass flux of different compositions of binary particle mixture.

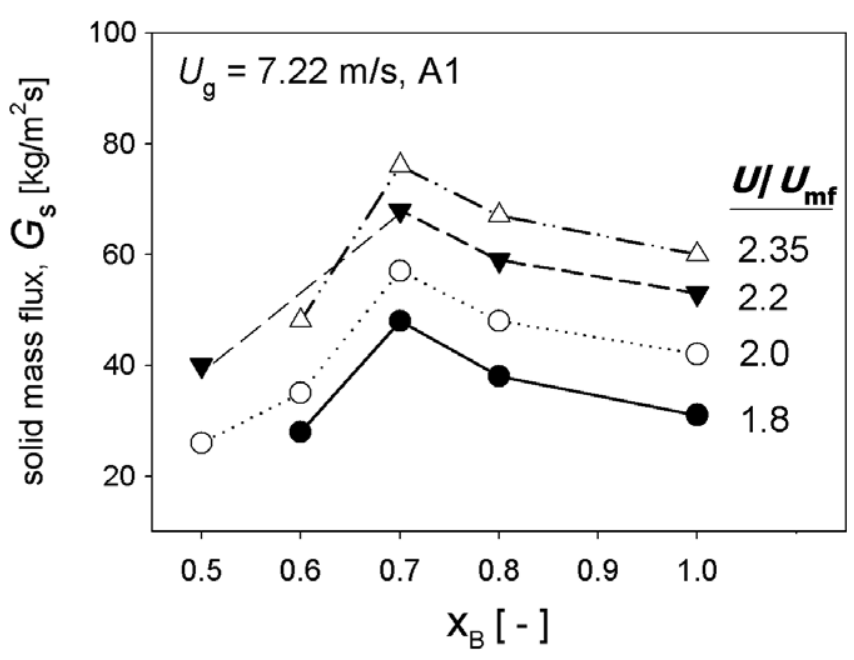

Fig. 14. $G_{\mathrm{s}}$ versus $x_{\mathrm{B}}$ for different $U / U_{\mathrm{mf}}$.

maximum and the minimum of $U / U_{\mathrm{mf}}$ ) increased as the fraction of the 194- $\mu \mathrm{m}$ sand particles $\left(\mathrm{x}_{\mathrm{B}}\right)$ was increased. Furthermore, at constant $U_{\mathrm{g}}$ and $U / U_{\mathrm{mf}}, G_{\mathrm{s}}$ of binary particle mixture reached a maximum value at $x_{\mathrm{B}}=0.7$, as shown in Fig. 14. The normalized energy of multi-scale pressure signals also showed significant dependency on the composition of binary particle mixture. As shown in Fig. $15 \mathrm{~b}, \bar{E}_{\text {meso }}$ in the horizontal part of L-valve revealed a similar dependency as $G_{\mathrm{s}}$ of the binary particle mixture on $x_{\mathrm{B}}$. It was explained that, for the $x_{\mathrm{B}}$-rich binary particle mixture $\left(x_{\mathrm{B}}=0.7,0.8\right.$ and 1.0), decreasing $x_{\mathrm{B}}$ or increasing $x_{\mathrm{D}}$ was helpful to reduce the shear stress between the pipe wall and the solid particles as the contact surface area was reduced. Hence, the solid particles were entrained through the L-valve more easily and the dune-ripple flow was enhanced in the horizontal part that caused $G_{\mathrm{s}}$ and $\bar{E}_{\text {meso }}$ to increase. However, on the other hand, decreasing $x_{\mathrm{B}}$ also reduced the contact surface area between the gas and solid phases that would cause the abatement of drag forces on
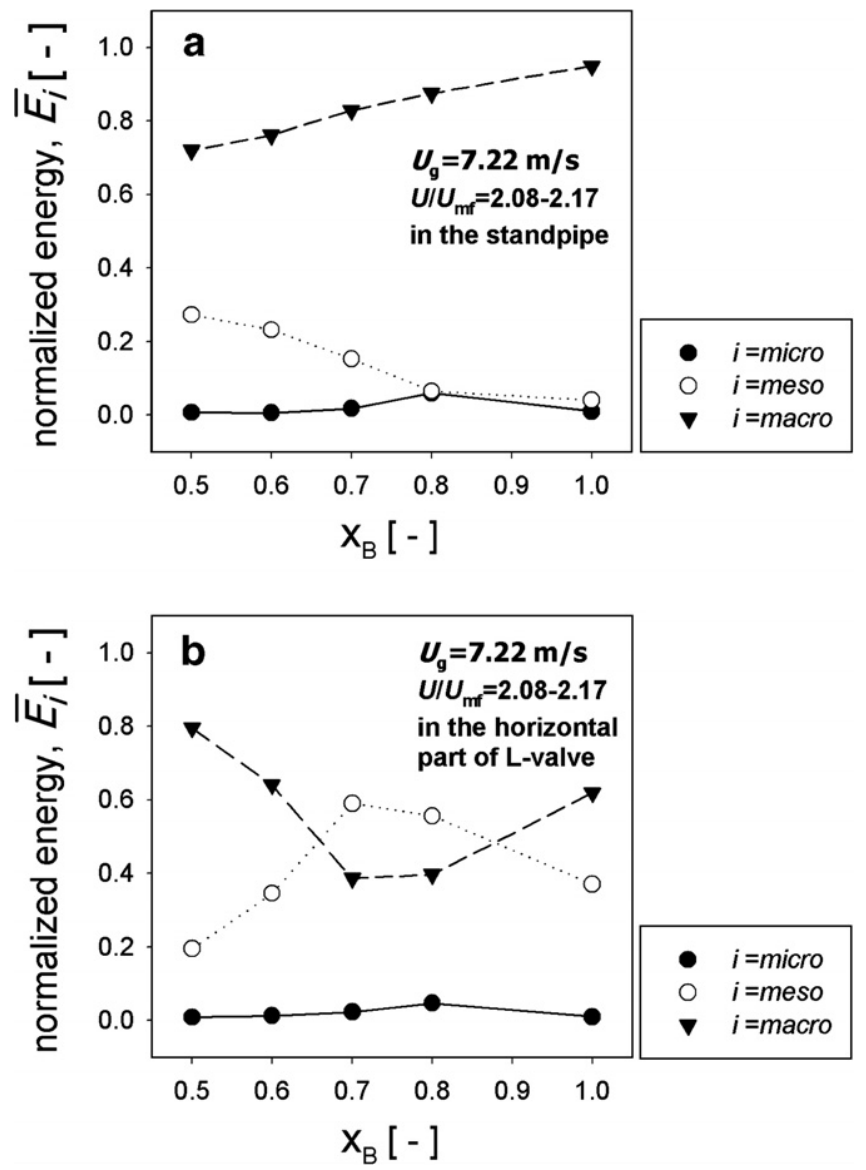

Fig. 15. Dependency of the normalized energy of micro-, meso- and macro-scale signals on the compositions of binary particle mixture.

the solid particles. Therefore $G_{\mathrm{s}}$ and $\bar{E}_{\text {meso }}$ decreased when $x_{\mathrm{B}}$ was below 0.7 . The variance of $\bar{E}_{\text {meso }}$ in Fig. 15 a could also elucidate the effect of the compositions on the performance of the standpipe. At approximately constant $U / U_{\mathrm{mf}}$, $\bar{E}_{\text {meso }}$ gradually increased with decreasing $x_{\mathrm{B}}$. It indicated that increasing the amount of the larger sand particles would increase the amount of gas flowing upwards along the standpipe. Further decreasing $x_{\mathrm{B}}$, the big bubbles/slugs even formed in the standpipe since the superficial gas velocity in the standpipe was higher than the incipient slugging velocity of the binary particle mixtures of $x_{\mathrm{B}}=0.5$ and 0.6. This result also agreed with Knowlton's (1997) observation. He described that if large particles are employed as bed material or when the solid flow rate in the standpipe is low, the aeration gas will flow upwards along the standpipe rather than be entrained by the solid particles flowing downwards. Consequently, the big bubbles/slugs are usually observed in the standpipe at these situations.

\section{Conclusions}

In this study, the flow dynamics of the six flow patterns was investigated by the pressure fluctuation signals measured at the standpipe and the horizontal part of the Lvalve. The multi-scale concept of MRA was applied to 
decompose the pressure fluctuations into micro-, meso- and macro-scale signals. The energy of these multi-scale signals had been reported to characterize the effects of operational parameters on the performance and pressure fluctuations of the L-valve. Several results had been concluded as follows,

- The energy of meso-scale signal $\bar{E}_{\text {meso }}$ at the standpipe increased apparently with heightening the aeration position. It represented bubbles formed in the standpipe and hindered the solid particles flowing through the L-valve especially at higher aeration rate.

- The energy of macro-scale signal $\bar{E}_{\text {macro }}$ at both the standpipe and the horizontal part of L-valve decreased with increasing the riser gas velocity. It implied that large slugs/cavities were gradually eliminated and the solid flow became smoother in the entire system.

- The dependency of $\bar{E}_{\text {meso }}$ on the composition of binary particle mixture $x_{\mathrm{B}}$ showed a similar trend as the solid mass flux $G_{\mathrm{s}}$ at the horizontal part of L-valve. Both $\bar{E}_{\text {meso }}$ and $G_{\mathrm{s}}$ reached a maximum value at $x_{\mathrm{B}}=0.7$.

Though the pressure fluctuation measurement indeed presented a convenient way for understanding the dynamic behavior of gas-solid flow in the L-valve, different kind of analytical tools including gamma or X-ray scan, CCD micro-camera, laser-sheet techniques, etc. should be used to re-confirm or check the flow regimes in the L-valve.

\section{References}

Arena, U., Langeli, C.B., Cammarota, A., 1998. L-valve behaviour with solids of different size and density. Powder Technol. 98, 231-240.

Cheung, L., Nienow, A.W., Rowe, P.N., 1974. Minimum fluidisation velocity of a mixture of different sized particles. Chem. Eng. Sci. 29, 1301-1303.
Geldart, D., Jones, P., 1991. The behavior of L-valve with granular powders. Powder Technol. 67, 163-174.

Jones, P.J., Leung, L.-S., 1985. Downflow of solids through pipes and valves. In: Davidson, J.F., Clift, R., Harrison, D. (Eds.), Fluidization. Academic Press, London, pp. 293-329.

Knowlton, T.M., Hirsan, I., 1978. L-valves characterized for solids flow. Hydrocarbon Proc. 57, 149-156.

Knowlton, T.M., 1997. Standpipes and return systems. In: Grace, J., Avidan, A.A., Knowlton, T.M. (Eds.), Circulating Fluidized Beds. Blackie Academic and Professional, New York, pp. 214-260.

Leung, L.S., Jones, P.J., 1978. Flow of gas-solid mixtures in standpipes. A review. Powder Technol. 20, 145-160.

Li, H., 2000. Multiresolution analysis of pressure fluctuation in horizontal swirling flow pneumatic conveying using wavelets. Adv. Powder Technol. 11, 423-438.

Li, H., 2002. Application of wavelet multi-resolution analysis to pressure fluctuations of gas-solid two-phase flow in a horizontal pipe. Powder Technol. 125, 61-73.

Loung, P.H., Bhattacharya, S.C., 1993. A study of solid circulation rate in a circulating fluidized bed. Int. J. Energy Res. 17, 479-490.

Lu, X., Li, H., 1999. Wavelet analysis of pressure fluctuation signals in a bubbling fluidized bed. Chem. Eng. J. 75, 113-119.

Mallat, S., 1989. A theory for multiresolution signal decomposition: the wavelet representation. IEEE Trans. Pattern Anal. Mach. Intell. 11, 674-693.

Ozawa, M., Tobita, S., Mii, T., Tomoyasu, T.Y., Takebayashi, T., Suzuki, K., 1991. Flow pattern and flow behavior of solid particles in L-valve. In: Basu, P., Horio, M., Hasatani, M. (Eds.), Circulating Fluidized Bed Technology III. Pergamon Press, Oxford, pp. 615-620.

Park, S.H., Kim, S.D., 2001. Wavelet transform analysis of pressure fluctuation signals in a three-phase fluidized bed. Korea J. Chem. Eng. 18, 1015-1019.

Ren, J., Li, J., 1998. Wavelet analysis of dynamic behavior in fluidized beds. In: Fan, L.-S., Knowlton, T.M. (Eds.), Proceedings of the Ninth Engineering Foundation Conference on Fluidization, Durango, Colorado, pp. 629-636.

Smolders, K., Baeyens, J., 1995. The operation of L-valves to control standpipe flow. Adv. Powder Technol. 6, 163-176.

Stewart, P.S.B., Davidson, J.F., 1967. Slug flow in fluidised beds. Powder Technol. 1, 61-80.

Wen, C.H., Yu, Y.H., 1966. A generalized method for predicting the minimum fluidization velocity. AIChE J. 12, 610-612. 